\title{
Alimentación en pacientes diagnosticados de insuficiencia renal crónica en tratamiento con diálisis
}

\author{
Alba GonZÁlez Gallego \\ al286552@uji.es \\ PAULA TARONGI VIDAL \\ al286598@uji.es \\ ELADIO JOAQUín COLLAdo BoIRA \\ colladoe@uji.es
}

\section{Resumen}

Introducción: La insuficiencia renal crónica (IRC) es una enfermedad progresiva que implica una pérdida gradual de los riñones para realizar sus funciones principales durante al menos tres meses. En las fases avanzadas, se realizan terapias de reemplazo renal, en las cuales se considera una parte fundamental el seguimiento de las recomendaciones alimentarias, y principalmente de las sustancias esenciales y agua. Como objetivo principal del trabajo se ha establecido conocer las recomendaciones alimentarias específicas para los pacientes diagnosticados de IRC con el fin de aumentar sus conocimientos, mejorar su cumplimentación y adhesión al tratamiento nutricional prescrito e incrementar su estado de salud y calidad de vida. Metodología: Se desarrolló una revisión integradora de la literatura, mediante la consulta de cuatro bases de datos científicas (Preevid, PubMed, BVS, Biblioteca Cochrane Plus) y el uso de dos páginas web oficiales. Se escogieron un total de cuatro artículos científicos, cinco guías de práctica clínica y tres resultados encontrados en las páginas webs oficiales. Resultados: La información utilizada muestra que la importancia de la alimentación en el tratamiento de la IRC, recomendando el inicio temprano, a través de un equipo multidisciplinar. Conclusiones: La alimentación en los pacientes con IRC es uno de los puntos clave en su tratamiento de remplazo de la función renal, con el fin de mejorar su salud y calidad de vida. Los profesionales enfermeros se consideran elementos imprescindibles en la educación para la salud, sin olvidar el aspecto psicológico.

Palabras clave: dieta, insuficiencia renal crónica, diálisis.

\footnotetext{
Abstract

Introduction: Chronic renal failure (CRF) is a progressive disease that involves a gradual loss of the kidneys' functions. Kidneys' alterations produces accumulations of waste products in the organism. During the later stages of the pathology, renal replacement therapies are used, in which is important to follow dietary recommendations, mainly of essential substances and water. The main objective is to know the specific
} 
dietary recommendations for patients diagnosed with CRF in order to increase their knowledge about the disease, improve their completion and adherence to the prescribed nutritional therapy and increase their state of health and quality of life. Methodology: An integrative review was carried out. A total of four databases were used (Preevid, Pubmed, BVS, Biblioteca Cochrane Plus), and two official web pages. A total of four scientific articles, five clinical practice guides and three results of official web pages were used to carry out the work. Results: The information shows the importance of diet in the CRF's treatment, recommended their early onset through multidisciplinary team. Conclusion: Nutrition is one of the key points in the treatment of chronic renal failure to maintain good health and quality of life. Nursing staff is considered a key element in health education, without forgetting their psychological aspect.

Keywords: diet, chronic renal insufficiency, renal dialysis.

\section{Introducción}

La insuficiencia renal crónica (IRC) es una enfermedad progresiva que implica una pérdida gradual de los riñones para realizar sus funciones principales durante al menos tres meses (Cantero et al., 2004, De las Heras, et al., 2015;). Es una enfermedad que alcanza una prevalencia en la población española relativamente elevada, afectando a casi el $10 \%$ de la población. Su incidencia aumenta con la edad, siendo de un $20 \%$ en personas mayores de 60 años y de un $35 \%$ en mayores de 70; y, en caso de padecer diabetes o enfermedad cardiovascular, aumenta hasta un $50 \%$.

El riñón es un órgano vital del cuerpo que se encarga de filtrar y limpiar la sangre de las toxinas y sustancias de desecho que se producen como consecuencia del metabolismo, y de regular el exceso de líquido (balance hidroelectrolítico) a través de la orina. Presenta también otras funciones imprescindibles: estimular la producción de glóbulos rojos de la sangre, regular la presión arterial y modificar sustancias como la vitamina D, dando fuerza a los huesos (Cantero et al., 2004; Diaverum, 2010; National Kidney Foundation, 2015). Cuando se produce un fallo en este órgano, aparece la IRC, generando un almacenamiento en el organismo de productos de desecho, acompañado de la sintomatología propia: náuseas, vómitos, diarreas, pérdida de apetito, cansancio, alteración del sueño, acidez o ardor de estómago, dolor de cabeza, decaimiento general, picor en la piel, etc. (Crespo et al., 2008).

En las fases más avanzadas, estados terminales de la patología, existen hasta tres opciones de terapia de remplazo renal: la hemodiálisis realizada a través de un acceso vascular (fístula o catéter central); la diálisis peritoneal en la que se emplea la membrana peritoneal para separar las sustancias; y, por último, el trasplante renal (Diaverum, 2010).

En los pacientes diagnosticados de IRC y durante cualquier tipo de diálisis, la alimentación presenta una gran relevancia (Hospital Universitario Reina Sofía, 2013; Hospital Universitario Donostia, 2013):

- Mejora la calidad de vida del paciente

- Permite que el resultado de la diálisis sea mucho más óptimo

- Mejora el estado de salud del paciente para un futuro trasplante renal. 
Por tanto, modificar la alimentación, no solo es una recomendación saludable sino que es parte fundamental del tratamiento de los pacientes diagnosticados de IRC (National Kidney Foundation, 2015). Para ello, es esencial la educación sanitaria y los cuidados enfermeros, destinados principalmente a mantener en niveles adecuados las sustancias esenciales para el organismo: proteínas, hidratos de carbono, minerales (sodio, potasio, fósforo y calcio) y agua (Crespo et al., 2008).

De este modo, se establece como objetivo general del presente trabajo: conocer las recomendaciones alimentarias específicas para los pacientes diagnosticados de Insuficiencia Renal Crónica con el fin de aumentar sus conocimientos, mejorar su cumplimentación y adhesión al tratamiento nutricional prescrito e incrementar su estado de salud y calidad de vida. Así mismo, los objetivos específicos son:

- Aumentar y mejorar los conocimientos que los pacientes con IRC presentan en relación a las proteínas, hidratos de carbono, minerales y líquido.

- Informar acerca de las complicaciones que conllevaría un desajuste en los niveles de las sustancias anteriores.

- Asesorar a los pacientes sobre los alimentos convenientes a consumir y los alimentos a evitar por su patología.

- Dar a conocer las recomendaciones relacionadas con una óptima preparación de los alimentos para mantener estables los niveles de dichas sustancias.

\section{Metodología}

Con el fin de obtener la mejor evidencia científica disponible sobre el tema planteado, se establecieron los DeCS (Descriptores en Ciencias de la Salud) «Dieta», «Insuficiencia Renal Crónica» y «Diálisis»; y los MESH (Medical Subject Headings) «Diet», «Renal insufficiency, chronic» y "Renal dialysis». A partir de estas palabras, se realiza una búsqueda de información científica útil, fiable y relevante en un total de cuatro bases de datos (Preevid, PubMed, Biblioteca Virtual en Salud (BVS), Biblioteca Cochrane Plus), mediante el uso de filtros: idioma español e inglés, texto completo disponible y últimos 5 años (2011-2016). Toda esta información relacionada con la estrategia de búsqueda se muestra en la Tabla 1.

Tabla 1

Estrategia de búsqueda por bases de datos (Fuente: Elaboración propia)

\begin{tabular}{ccc}
\hline Base de datos & Estrategia de búsqueda & Resultados \\
\hline Preevid & «Insuficiencia renal crónica» & 37 \\
& «Diálisis» & 25 \\
"Dieta» & 154 \\
BVS & «Renal insufficiency, chronic AND Renal dialysis AND & 31 \\
Diet» & "Insuficiencia renal crónica AND Diálisis AND Dieta» & 138 \\
Biblioteca Cochrane Plus & «Insuficiencia renal crónica AND Diálisis AND Dieta» & 1 \\
\hline
\end{tabular}


Por último, ha sido utilizada tanto la página web de la Fundación Renal Española como la página web Norte-Americana de la Fundación Nacional del Riñón dedicada a la sensibilización, prevención y tratamiento de la enfermedad renal.

\section{Resultados}

Tras la realización de la búsqueda en las diferentes bases de datos, se utilizaron un total de cuatro artículos científicos (1 de Preevid, 2 de PubMed y 1 de Biblioteca Cochrane Plus). Así mismo, se utilizaron unas guías de práctica clínica y diferentes resultados de las páginas web oficiales comentadas anteriormente.

En todos ellos, la alimentación se considera uno de los puntos clave en el tratamiento de la Insuficiencia Renal Crónica. Aunque en general no se especifica la etapa concreta de la enfermedad en el que se deben empezar las intervenciones de educación dietética/nutricional, sí se recomienda que comience de forma temprana y basada en información de los diferentes nutrientes (proteínas, hidratos de carbono, sodio, potasio, fósforo, calcio y líquido) (Murciasalud, 2015; D'Alessandro et al., 2015).

Las proteínas se encargan de la construcción y renovación de células y tejidos, y de combatir infecciones (Crespo $\mathrm{R}$ et al., 2008). De forma general, la ingesta recomendada para un paciente tratado con diálisis es de 1-1,2 $\mathrm{g} / \mathrm{kg}$ de peso por día, debiendo ser aproximadamente el $50 \%$ de elevado valor biológico.

Los hidratos de carbono son el único grupo de alimentos cuyo consumo no está limitado en los pacientes en tratamiento con diálisis, salvo si el paciente presenta un diagnóstico de diabetes. Debe recomendarse consumir hidratos de carbono complejos y evitar los simples, los cuales favorecen el incremento de los valores de colesterol, triglicéridos, obesidad, etc. (Hospital Universitario Reina Sofía, 2013; Hospital Universitario Donostia, 2013).

Los valores elevados de sodio en el cuerpo son los responsables del aumento de la tensión arterial, el aumento de peso, edemas en piernas, cara, ojos, llegando incluso a la insuficiencia cardiaca y al edema de pulmón (National Kidney Foundation, 2015; Cresp et al., 2008; Hospital Universitario Reina Sofía, 2013). Durante el tratamiento con diálisis, debe seguirse una dieta pobre en sal, recomendando un consumo entre 1.300 y 1.700 mg. diarios. Para su consecución, entre las recomendaciones encontramos (Hospital Universitario Donostia, 2013; National Kidney Foundation):

- Evitar la utilización de sal de mesa. Utilizar otras especias o zumo de limón que dan sabor a los platos y los hace más apetecibles.

- Comer pan sin sal.

- No utilizar concentrados para carnes o pescados, ni sopas de sobre.

En relación al potasio, éste se puede acumular en la sangre pudiendo provocar alteraciones en la actividad muscular, calambres, hormigueos, debilidad, arritmias e incluso parada cardiaca (National Kidney Foundation). Con el fin de que los pacientes consigan reducir las cantidades de potasio, consumiendo entre 1.800 y $2.000 \mathrm{mg}$ al día, pueden ser proporcionadas una serie de recomendaciones (Diaverum, 2010; Crespo et al., 2008; Hospital Universitario Reina Sofía, 2013; Hospital Universitario Donostia, 2013):

- Ingerir como máximo una pieza de fruta cruda al día y dejarla en remojo como mínimo entre tres y ocho horas, cambiando el agua en varias ocasiones. 
- Cocer la fruta para reducir la cantidad de potasio hasta la mitad.

- Las verduras y hortalizas deberán ser hervidas una o dos ocasiones con abundante agua, tirando ésta a mitad de cocción y repitiendo la operación.

- Las setas deben consumirse como máximo cada quince días y las legumbres dos veces a la semana, medidas en un plato de café y cocinarlas con el proceso de doble ebullición.

- Desechar el jugo de las conservas ya que presenta gran cantidad de potasio.

- Consumir la carne en estofados o en salsas, dado que pierde la mitad de potasio si se desecha el agua de cocción.

- Hacer la salsa de tomate casera, con tomate natural escaldado o con tomate envasado entero, eliminando la piel y el jugo.

- No utilizar levaduras para los rebozados o repostería.

Tanto el fósforo como el calcio, son el principal componente de los huesos (National Kidney Foundation). Un exceso de fósforo en la sangre produce una salida de calcio de los huesos, quedando éstos más frágiles (National Kidney Foundation, 2015). El consumo recomendado de fósforo se encuentra entre 800 y $1.200 \mathrm{mg}$.; mientras que el de calcio entre 1.400 y $1.600 \mathrm{mg}$ al día. Entre las recomendaciones dietéticas a seguir, encontramos (Crespo et al., 2008; Hospital Universitario Donostia, 2013):

- Consumir productos lácteos enteros, evitando los desnatados. Se debe tomar como máximo un vaso de leche al día. Fomentar el consumo de leche de soja y de almendras, ya que están compuestas de una menor cantidad de fósforo.

- No consumir más de dos o tres yogures, flanes, mousses o cuajadas, o $100 \mathrm{~g}$ de requesón o queso fresco a la semana y tomar como máximo dos huevos.

- Aumentar la ingesta de pescado blanco.

Además, durante el tratamiento de diálisis, el paciente debe controlar la cantidad de líquidos que consume para evitar complicaciones como: tensión arterial elevada, edemas, dificultad para respirar y calambres y mareos durante la sesión de diálisis. Este concepto de «líquidos» no sólo se incluye el agua, sino también los aquellos alimentos con un alto contenido en ésta. Como regla práctica, se debe recomendar consumir $500 \mathrm{ml}$ de líquido más de los que el paciente orina en 24 horas. Entre las pautas a seguir encontramos (Diaverum, 2010; Crespo et al., 2008; Hospital Universitario Reina Sofía, 2013; Hospital Universitario Donostia, 2013):

- Medir la cantidad de líquido total que debe consumir al día, teniendo en cuenta situaciones especiales como la fiebre, diarrea o sudoración importante.

- Eliminar el agua de las verduras salteándolas después de hervirlas.

- Consumir pan tostado, ya que presenta un $35 \%$ menos de agua.

- Utilizar rodajas de limón para estimular la salivación y reducir la sensación de sed, limitando su consumo a dos rodajas al día, ya que el limón contiene potasio. Además, se puede consumir chicles y caramelos de menta.

- Remplazar las bebidas por cubitos de hielo en los días más calurosos.

- Limitar la ingesta de comidas saladas que aumentan la sensación de sed.

Por último, con el fin de establecer una pequeña diferencia entre la hemodiálisis y la diálisis peritoneal, cabe destacar que los pacientes tratados con esta última pueden 
disfrutar de una mayor libertad en la dieta, ya que al no existir periodos interdiálisis tan prolongados no se acumulan en el organismo tantos productos tóxicos. Sin embargo, deben seguir las mismas recomendaciones en lo que se refiere a los alimentos previamente expuestos y sus componentes (Crespo et al., 2008)

\section{Discusión y conclusiones}

Como se puede observar con la información, la alimentación en los pacientes diagnosticados de IRC es uno de los puntos clave e imprescindibles en su tratamiento de remplazo renal, con el fin de mantener un buen estado de salud y calidad de vida.

Para alcanzar estos objetivos, y considerando a los profesionales enfermeros como elementos claves en la educación para la salud, es esencial proporcionar a los pacientes los conocimientos necesarios y suficientes con el fin de capacitarlos para seleccionar aquellos alimentos que son adecuados para su patología, las técnicas más óptimas para una correcta preparación y consumición; así como detectar y evitar el consumo de aquellos alimentos inadecuados y perjudiciales para su salud.

Como profesionales, y de forma multidisciplinar, consideramos imprescindible realizar una valoración inicial tanto del paciente como de los familiares o cuidadores, con el fin de valorar la capacidad de compresión y detectar los recursos de éstos para seguir todas las recomendaciones. Éstas deberán ser explicadas con ejemplos gráficos de fácil comprensión, suprimiendo las recomendaciones complejas en forma de gramos o miligramos.

Por último, no debemos olvidar el aspecto psicológico del paciente, ni el estrés y ansiedad que conlleva el seguimiento de una dieta tan estricta. De esta forma, los familiares deben ser involucrados en el proceso de la enfermedad y su tratamiento, intentando que el cumplimiento de los consejos dietéticos no aísle al paciente a la hora de realizar las comidas; instruyendo de que, con pequeñas modificaciones, la dieta puede ser satisfactoria para todos los componentes de la familia.

\section{Referencias bibliográficas}

Cantero, P. \& Ruano, A. (2009). Eficacia y efectividad del inicio precoz del tratamiento renal sustitutivo en la insuficiencia renal crónica avanzada. Recuperado en abril 25, 2016 de: http://www.bibliotecacochrane.com/BCPGetDocument.asp?SessionID=\%2010553771 \&DocumentID=AEG000045

Crespo, R., Casas, R. \& Contreras MD. (2008). Guía para el paciente renal según modalidades de tratamiento ( $1^{\text {a }}$ edición). Córdoba: Alcer-Córdoba.

D’Alessandro C., Piccoli, G. B. \& Cupisti, A. (2015). The «phosphorus pyramid»: a visual tool for dietary phosphate management in dialysis and CKD patients. BMC Nephrolog, 16, $1-6$.

De las Heras, M. T. \& Martínez, C. (2015). Conocimiento y percepción nutricional en diálisis: su influencia en la transgresión y adherencia. Estudio inicial. Nutrición Hospitalaria, 31, 1366-1375.

Diaverum (2010). Manual Informativo para el paciente renal ( $2^{a}$ edición). Madrid.

Fundación renal. (2013). Disponible en: https://www.friat.es 
Hospital Universitario Donostia: Unidad de Nefrología-Diálisis (2013). Guía de Alimentación en pacientes con Insuficiencia Renal (1 ${ }^{a}$ edición). Donostia: Unidad de Comunicación del Hospital Universitario Donostia.

Hospital Universitario Reina Sofía. Servicio de Nefrología. (2013). Guía para pacientes con tratamiento de hemodiálisis ( $1^{a}$ edición). Córdoba: Alcer-Córdoba.

Murciasalud. (2015) ¿Con qué grado de insuficiencia renal hay que comenzar la educación dietética renal para una correcta prevención? Recuperado en abril 25, 2016 de: https:// www.murciasalud.es/preevid.php?op=mostrar_pregunta\&id=20357\&idsec=453

National Kidney Foundation. New York. Disponible en: https://www.kidney.org/

National Kidney Foundation (2015). Nutrición e insuficiencia renal crónica. Abril 25, 2016, de National Kidney Foundation, Sitio web: https://www.kidney.org/sites/default/files/docs/1150-1105_aai_patbro_nutritionandckd_3-5_pharmanet_nkf_span_jan08.pdf 